POLLACK PERIODICA

An International Journal for Engineering and Information Sciences

DOI: $10.1556 / 606.2020 .15 .2 .9$

Vol. 15, No. 2, pp. 94-105 (2020)

www.akademiai.com

\title{
MODELING AND ROBOTIC HANDLING OF A SNAP-FITTING BOX BUCKLE
}

\author{
${ }^{1}$ László RÓNAI*, ${ }^{2}$ Tamás SZABÓ \\ ${ }^{1,2}$ Robert Bosch Department of Mechatronics, Faculty of Mechanical Engineering and \\ Informatics, University of Miskolc, Miskolc-Egyetemváros, 3515, Hungary \\ e-mail: ${ }^{1}$ ronai.laszlo@uni-miskolc.hu, ${ }^{2}$ szabo.tamas@uni-miskolc.hu
}

Received 2 October 2019; accepted 25 October 2019

\begin{abstract}
Modeling and robotic handling of a plastic box buckle is discussed in this paper. The closing mechanism of the box buckle is simulated to determine the characteristic of the nonlinear load-deflection curve. An intelligent end-effector was designed and manufactured to handle the assembly with a robot. The closing force is measured by a built-in load cell and its values are processed by a micro-controller. The intelligent end-effector can be used in a robotic system, which deals with different snap-fit applications.
\end{abstract} effector

Keywords: Robotic assembly, Snap-fit, Nonlinear finite element method, Intelligent end-

\section{Introduction}

The human haptic concept is important for numerous manual assembly operations [1]. Nowadays more and more companies change from the manual assembly to the automated one, which can be carried out by e.g. industrial robots. In general, industrial robots cannot provide haptic feedback [1]. Position-based assembly with industrial robots may raise assembly uncertainties [2]. Besides prescribed positions, it is a key feature to measure assembly forces.

Although commercial force sensors for robotic operations are available [3] but these sensors are relative expensive and can provide only restricted programming capability for tasks, which may have loss of stability. In this paper an intelligent end-effector is

\footnotetext{
${ }^{*}$ Corresponding Author
} 
applied to find the releasing point of e.g. snap-fit joints [4], [5]. The load-deflection curve of most of the snap-fit elements contains an unstable branch. There are several approaches to develop an end-effector, which contains load cells [6], [7].

The main purpose of this paper is modeling and simulating the closing process of a plastic box buckle using nonlinear Finite Element Method (FEM), then to compare the results obtained via an industrial robot. An intelligent end-effector has been developed, which contains a load cell to measure the forces during the assembly operation and a Micro-Controller (MC), which processes the measured data in order to determine snapthrough point.

\section{Modeling and simulation of the closing process}

A plastic buckle of a box is shown in Fig. 1 and its mechanical model can be seen in Fig. 2. The buckle element consists of three joints A, F, G. The curved beam has a radius $R=70 \mathrm{~mm}$. Angle $\varphi_{1}$ and $\varphi_{2}$ of the curved beam D-E are equal to $2.35 \mathrm{rad}$ and $0.93 \mathrm{rad}$, respectively. The beam structure is subdivided to 12 straight $2 \mathrm{D}$ beam FEM elements and it contains 13 nodes. The point $\mathrm{C}$ is on the wall of the box where the buckle may have contact, in the initial configuration there is a gap $h$ between point $\mathrm{B}$ and point $\mathrm{C}$.

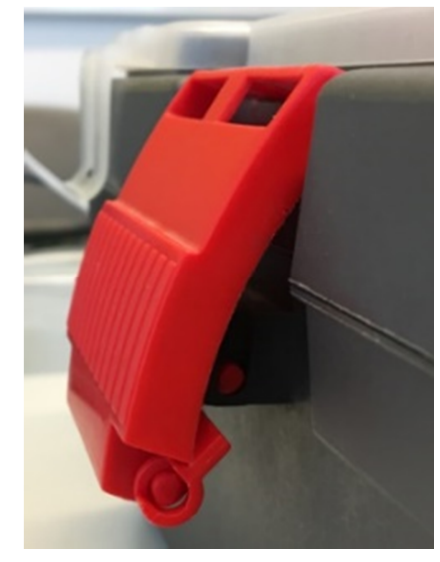

Fig. 1. The plastic box buckle

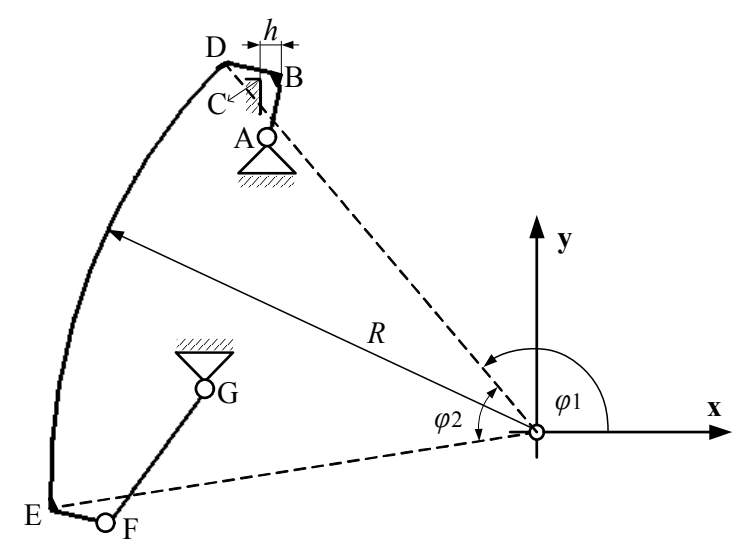

Fig. 2. The model of the plastic buckle

The beam elements may suffer large displacements and rotations, small strains without shear deformations. These structures can be modeled with co-rotational approach or total Lagrangian description [8]. A beam element is shown in Fig. 3 in two configurations, i.e. the initial and current configurations. The previous one has an angle $\beta_{0}$ and the later one has an angle $\alpha$, which is measured from the initial configuration. The deformed configuration is given by the angle $\beta$. The global nodal rotations of the first and second node are denoted by $\Theta_{1}$ and $\Theta_{2}$. The local nodal rotations $\Theta_{1 l}, \Theta_{2 l}$ due to deformation can be given in a local coordinate system $X_{l}, Y_{l}$. 


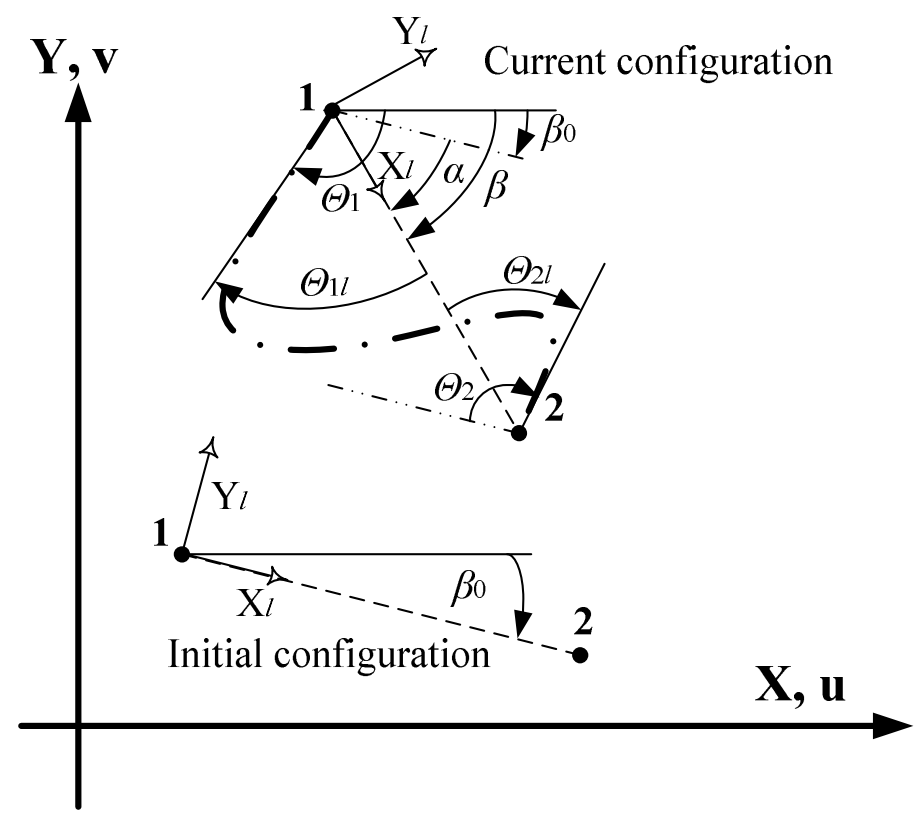

Fig. 3. Definition of the initial and current configurations

Souza published a formula for the local nodal rotations to determine its value without any limitation [9]:

$$
\begin{aligned}
& \Theta_{1 l}=\arctan \left(\frac{\sin \left(\Theta_{1 l}\right)}{\cos \left(\Theta_{1 l}\right)}\right)=\arctan \left(\frac{\cos (\beta) \sin \left(\beta_{1}\right)-\sin (\beta) \cos \left(\beta_{1}\right)}{\cos (\beta) \cos \left(\beta_{1}\right)+\sin (\beta) \sin \left(\beta_{1}\right)}\right) \\
& \Theta_{2 l}=\arctan \left(\frac{\sin \left(\Theta_{2 l}\right)}{\cos \left(\Theta_{2 l}\right)}\right)=\arctan \left(\frac{\cos (\beta) \sin \left(\beta_{2}\right)-\sin (\beta) \cos \left(\beta_{2}\right)}{\cos (\beta) \cos \left(\beta_{2}\right)+\sin (\beta) \sin \left(\beta_{2}\right)}\right),
\end{aligned}
$$

where $\beta_{1}=\Theta_{1}+\beta_{0}, \beta_{2}=\Theta_{2}+\beta_{0}$

A local internal element load vector $\overline{\mathbf{f}}_{i}^{e}$ contains axial forces $N_{i}, N_{j}$, end moments $M_{i}, M_{j}$ of the beam and shear forces $V_{i}, V_{j}$. A nodal displacement vector $\overline{\mathbf{q}}^{e}$ consists of axial nodal displacements $\bar{u}_{i}, \bar{u}_{j}$ lateral nodal displacements $\bar{v}_{i}, \bar{v}_{j}$ and nodal rotations $\bar{\Theta}_{i}, \bar{\Theta}_{j}$. A tangent stiffness matrix $\overline{\mathbf{K}}_{t}^{e}$ of one element is the sum of a local linear $\overline{\mathbf{K}}_{L}^{e}$ and a local geometric stiffness matrix $\overline{\mathbf{K}}_{g}^{e}$ : 


$$
\overline{\mathbf{K}}_{t}^{e}=\overline{\mathbf{K}}_{L}^{e}+\overline{\mathbf{K}}_{g}^{e}
$$

The linear geometric stiffness matrix is given by

$$
\overline{\mathbf{K}}_{L}^{e}=\left[\begin{array}{cccccc}
\frac{A E}{L_{0}} & 0 & 0 & -\frac{A E}{L_{0}} & 0 & 0 \\
0 & \frac{12 E I}{L_{0}^{3}} & \frac{6 E I}{L_{0}^{2}} & 0 & -\frac{12 E I}{L_{0}^{3}} & \frac{6 E I}{L_{0}^{2}} \\
0 & \frac{6 E I}{L_{0}^{2}} & \frac{4 E I}{L_{0}} & 0 & -\frac{6 E I}{L_{0}^{2}} & \frac{2 E I}{L_{0}} \\
-\frac{A E}{L_{0}} & 0 & 0 & \frac{A E}{L_{0}} & 0 & 0 \\
0 & -\frac{12 E I}{L_{0}^{3}} & -\frac{6 E I}{L_{0}^{2}} & 0 & \frac{12 E I}{L_{0}^{3}} & -\frac{6 E I}{L_{0}^{2}} \\
0 & \frac{6 E I}{L_{0}^{2}} & \frac{2 E I}{L_{0}} & 0 & -\frac{6 E I}{L_{0}^{2}} & \frac{4 E I}{L_{0}}
\end{array}\right],
$$

where $A$ is the area of the cross section, $I$ is the area moment of inertia, $E$ is the Young's modulus and $L_{0}$ is the length of the beam element.

$$
\overline{\mathbf{K}}_{g a}^{e}=\frac{A E}{L_{0}} \frac{u l}{L_{0}}\left[\begin{array}{cccccc}
1 & 0 & 0 & -1 & 0 & 0 \\
0 & \frac{6}{5} & \frac{L_{0}}{10} & 0 & -\frac{6}{5} & \frac{L_{0}}{10} \\
0 & \frac{L_{0}}{10} & \frac{2 L_{0}^{2}}{15} & 0 & -\frac{L_{0}}{10} & -\frac{L_{0}^{2}}{30} \\
-1 & 0 & 0 & 1 & 0 & 0 \\
0 & -\frac{6}{5} & -\frac{L_{0}}{10} & 0 & \frac{6}{5} & -\frac{L_{0}}{10} \\
0 & \frac{L_{0}}{10} & -\frac{L_{0}^{2}}{30} & 0 & -\frac{L_{0}}{10} & \frac{2 L_{0}^{2}}{15}
\end{array}\right] .
$$

The geometric stiffness matrix can be produced with different ways; one of them is to develop the geometric stiffness matrix (5) from the displacement approximation based on the interpolation functions and the principles of the virtual displacement [10]. The second term of the internal virtual work, which contains the nonlinear increment of the strain, can be used to formulate the geometric stiffness matrix. 
Another way is to build the matrix based on virtual work calculated by the nodal internal forces and nodal virtual displacements [8], as it is shown in the next equation, where $u_{l}$ is the local axial displacement,

$$
\overline{\mathbf{K}}_{g b}^{e}=\left[\begin{array}{cccccc}
0 & \frac{M_{2}+M_{1}}{L_{0}^{2}} & 0 & 0 & -\frac{M_{2}+M_{1}}{L_{0}^{2}} & 0 \\
\frac{M_{2}+M_{1}}{L_{0}^{2}} & \frac{A E u_{l}}{L_{0}^{2}} & 0 & -\frac{M_{2}+M_{1}}{L_{0}^{2}} & -\frac{A E u_{l}}{L_{0}^{2}} & 0 \\
0 & 0 & 0 & 0 & 0 & 0 \\
0 & -\frac{M_{2}+M_{1}}{L_{0}^{2}} & 0 & 0 & \frac{M_{2}+M_{1}}{L_{0}^{2}} & 0 \\
-\frac{M_{2}+M_{1}}{L_{0}^{2}} & -\frac{A E u_{l}}{L_{0}^{2}} & 0 & \frac{M_{2}+M_{1}}{L_{0}^{2}} & \frac{A E u_{l}}{L_{0}^{2}} & 0 \\
0 & 0 & 0 & 0 & 0 & 0
\end{array}\right] .
$$

Before assembling the elements transformation $\mathbf{T}_{e}^{T}$ is applied to obtain the global element nodal forces and stiffness matrices into Cartesian coordinate system:

$$
\mathbf{f}_{i}^{e}=\mathbf{T}_{e}^{T} \overline{\mathbf{f}}_{i}^{e}, \quad \mathbf{K}_{t}^{e}=\mathbf{T}_{e}^{T} \overline{\mathbf{K}}_{t}^{e} \mathbf{T}_{e}
$$

The vector of the loads and the stiffness matrix of the complete structure are written as

$$
\mathbf{f}_{i}=\sum_{e} \mathbf{f}_{i}^{e}, \quad \mathbf{K}_{t}=\sum_{e} \mathbf{K}_{t}^{e}
$$

Newton-Raphson iteration $s=1,2,3, \ldots$, method is used to determine the nodal displacement vector:

$$
{ }^{t+\Delta t} \mathbf{q}^{(s)}={ }^{t+\Delta t} \mathbf{q}^{(s-1)}+\Delta \mathbf{q}^{(s)},
$$

where $\Delta \mathbf{q}^{(s)}$ consists the increment of the nodal point displacements.

$$
{ }^{t+\Delta t} \mathbf{K}_{t}^{(s)} \Delta \mathbf{q}^{(s)}={ }^{t+\Delta t} \mathbf{f}_{u}^{(s)}{ }^{t+\Delta t} \mathbf{f}_{i}^{(s-1)}
$$

where ${ }^{t+\Delta t} \mathbf{f}_{u}^{(s)}$ is the vector of the kinematical load in iteration step $s$ at time $t+\Delta t$. The iteration is terminated when the relative error of the unbalanced loads is smaller than a prescribed value $\varepsilon$. 
$\varepsilon=\frac{\| t+\Delta t \mathbf{f}_{u}^{(s)} \_t+\Delta t}{\mathbf{f}_{i}^{(s-1)} \|} \| 10^{-8}$.

\subsection{Simulation results}

A special purpose FEM program has been developed under Scilab program system. The program can handle elastic flexible $2 \mathrm{D}$ beam structures undergoing large displacements and rotations but small deformations, friction is not considered.

The material of the plastic buckle is PolyPropylene (PP) in Fig. 2, which has a Young's modulus $E=1300 \mathrm{MPa}$. There are three different structural components with different beam properties. The geometric properties of the first and the second beam component are given as area $A_{\mathrm{A}-\mathrm{B}}=A_{\mathrm{B}-\mathrm{D}}=120 \mathrm{~mm}^{2}$, inertia $I_{\mathrm{A}-\mathrm{B}}=I_{\mathrm{B}-\mathrm{D}}=40 \mathrm{~mm}^{4}$. The third beam component is given by $A_{\mathrm{D}-\mathrm{E}}=142 \mathrm{~mm}^{2}, I_{\mathrm{D}-\mathrm{E}}=178 \mathrm{~mm}^{4}$. The forth and the fifth component has the same cross section with $A_{\mathrm{E}-\mathrm{F}}=A_{\mathrm{F}-\mathrm{G}}=36 \mathrm{~mm}^{2}, I_{\mathrm{E}-\mathrm{F}}=I_{\mathrm{F}-\mathrm{G}}=12 \mathrm{~mm}^{4}$.

The solution of (10) is performed by displacement control. The horizontal displacement of structural point $\mathrm{E}$ is increased in 40 steps with uniform increments $\Delta u_{\mathrm{E}}=0.5 \mathrm{~mm}$. The displacements of the structure determined by simulation are shown in Fig. 4. The initial position shown with thin solid line is the left one, only every fifth step is drawn for the better visibility. The buckle elements with thick solid lines represent the case of positive assembly force. The negative values of the assembly forces have been obtained in the positions drawn by dash-dotted lines.

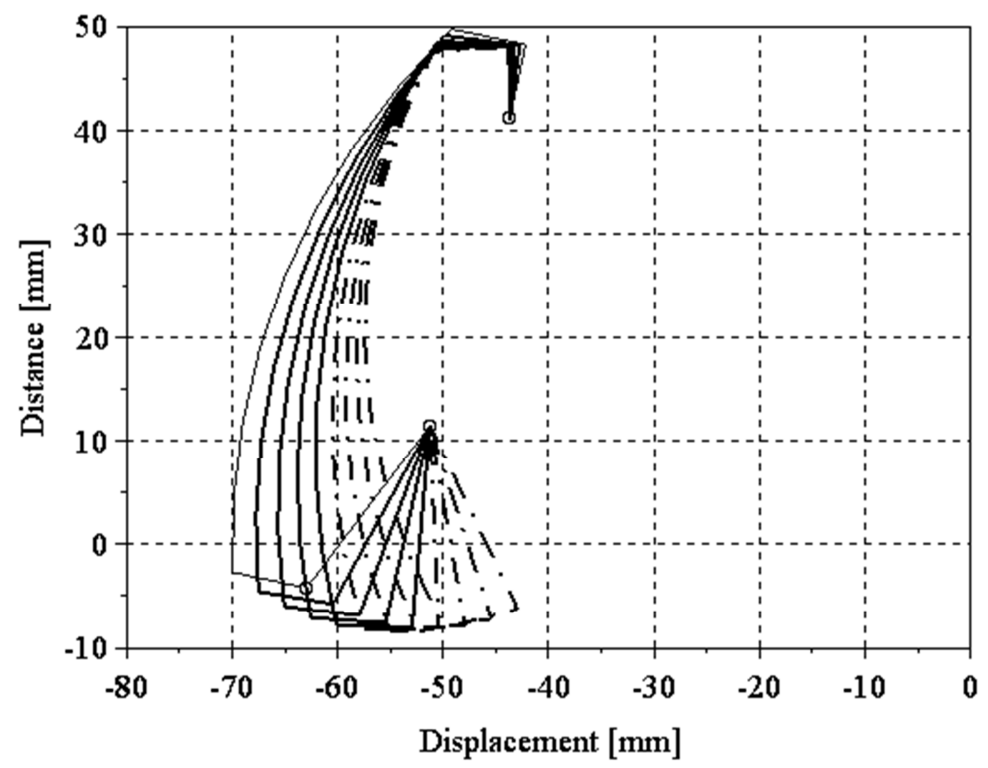

Fig. 4. Every fifth step is shown 
The assembling force $F_{a}$ is shown in Fig. 5, where the solid line represents the positive values and the dashed line shows its negative values. Displacement is prescribed horizontally at point $\mathrm{E}$ in the model. However, in the robotic assembly it is performed by a unilateral contact between the gripper and the buckle in point $\mathrm{E}$. Physical contact occurs only along the solid line. Then snap-fit takes place at the mutual border between the solid and dashed line, which is denoted by a small circle. Thereafter the buckle is closed autonomously. The maximum value of the contact force is $F_{a}=4 \mathrm{~N}$.

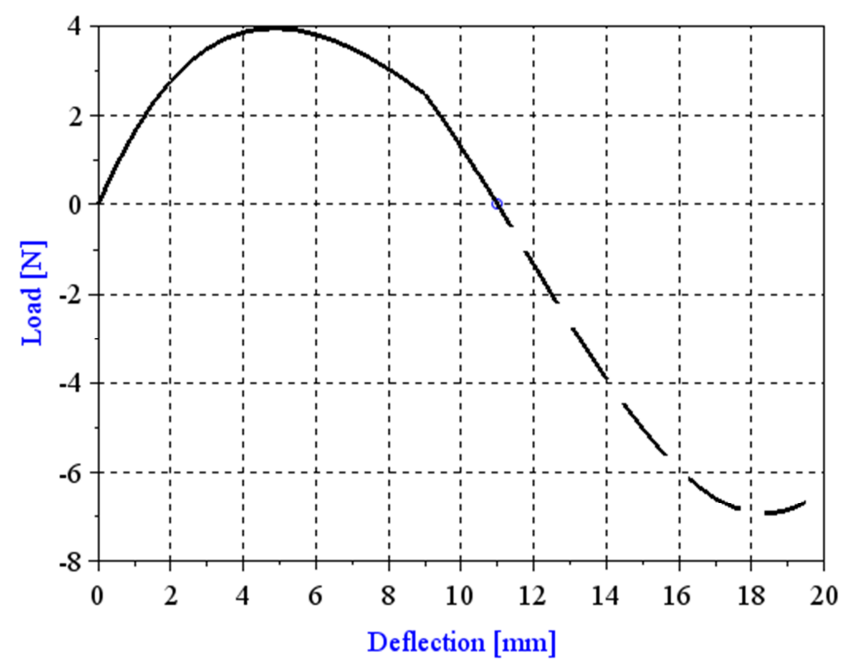

Fig. 5. The load-deflection curve of the plastic buckle

The simulation has been performed with the two different geometric stiffness matrices given in (5), (6) with the same tolerance $\varepsilon=10^{-8}$ to terminate the equilibrium iteration in (11). The number of iteration steps was smaller for with a consistent geometric stiffness matrix given in (5) comparing to its counterpart (6) obtained by corotational method. It was experienced that in every load increment the number of equilibrium iteration steps for the consistent geometric stiffness matrix was less with 1 comparing to the co-rotational method. It means that the convergence of the consistent method is better with $10 \%$ than the other one.

\section{Robotic assembly with an intelligent end-effector}

An intelligent end-effector has been developed and manufactured in order to treat assembling tasks, which may have loss of stability. An AVR type MC with an A/D converter, a load cell and a self-devised Printed Circuit Board (PCB) are the main electronic elements of the system.

The maximum capacity of the load cell is $200 \mathrm{~N}$ and 4 strain gages are glued on it. The assembling force is determined by the signals of the strain gages. Fig. 6 shows the $\mathrm{A} / \mathrm{D}$ converter, the PCB and the MC, which is soldered on an Arduino Nano platform. 
The communication between the robot and the $\mathrm{MC}$ carried out via digital input and output channels. Opto-couplers are mounted onto the self-devised PCB since the voltage level is different between the $\mathrm{MC}$ and the robot.

The test bench of the closing process of the box buckle is shown in Fig. 7. The end effector unit besides the electronic units contains a parallel mechanism, which can eliminate disturbing force and torque components [11]. Furthermore, an overload protection with setscrews was built in. The system can communicate with a laptop through USB port and it is capable to store the measured assembling forces.

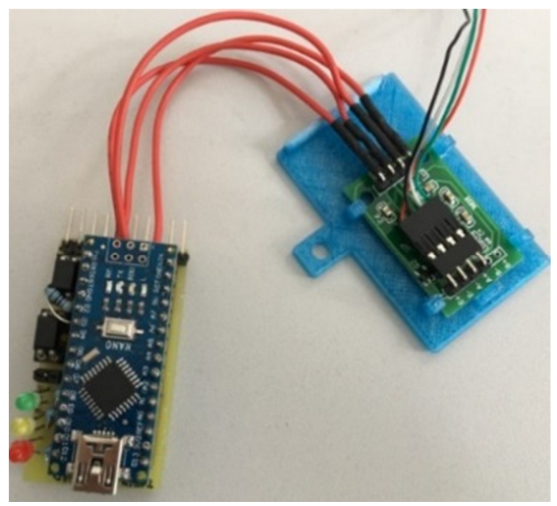

Fig. 6. The electronic elements of the intelligent end-effector

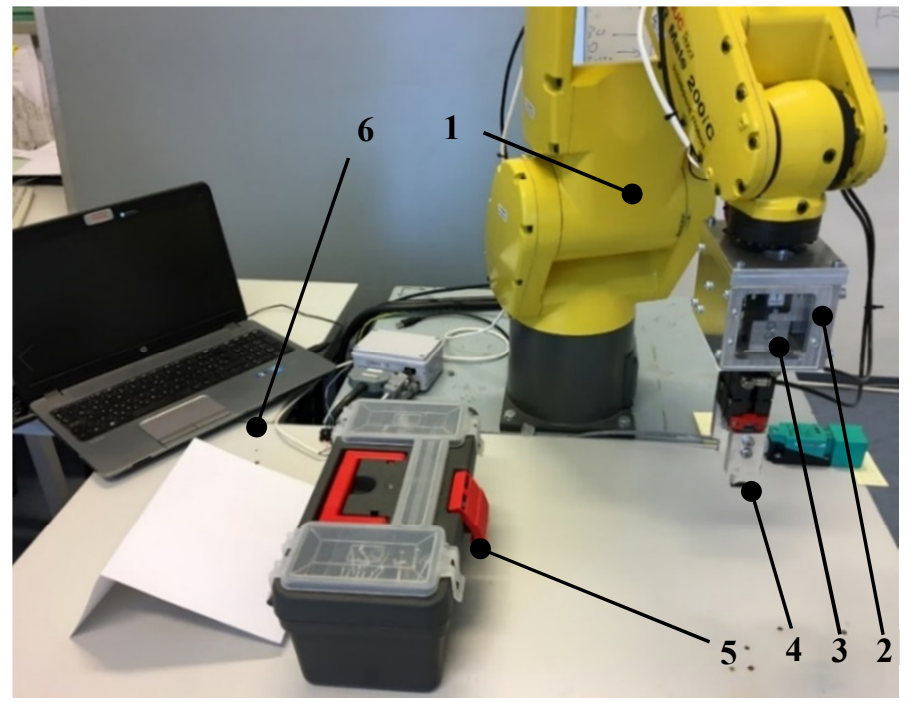

Fig. 7. The testbench: 1-Fanuc industrial robot; 2-intelligent end effector; 3-load cell; 4-pneumatic gripper; 5-plastic box buckle; 6-laptop 
A pneumatic gripper is mounted on the intelligent end-effector to make possible manipulation of work pieces. The tests have been carried out with a Fanuc LRMate 200iC type industrial robot, its controller is not an open-access one.

The robot starts the assembling process from a base position then it moves the end effector close to the buckle. The controller sends a digital signal to the MC to set zero value of the load cell. The MC starts to measure the forces. A loop in the robot program provides the $0.2 \mathrm{~mm}$ displacement increments. The end-effector is pushing the plastic buckle while the assembly force is measured.

The event of the snap-through phenomenon is determined by the MC with an algorithm. The MC sends a signal to the digital input of the robot to stop the loop of displacement increments. Thereafter the robot moves the base position and a signal is sent to the MC to set the initial values of the variables.

\subsection{Algorithm of the microcontroller}

In order to determine the snap-through phenomenon the algorithm takes into consideration the characteristics of the load-deflection curve, which can be the maximum and average values of the force function and detection its negative gradient or slope. The termination point usually is located on the downhill side of the loaddeflection curve (see Fig. 8) and the force value is close to zero.

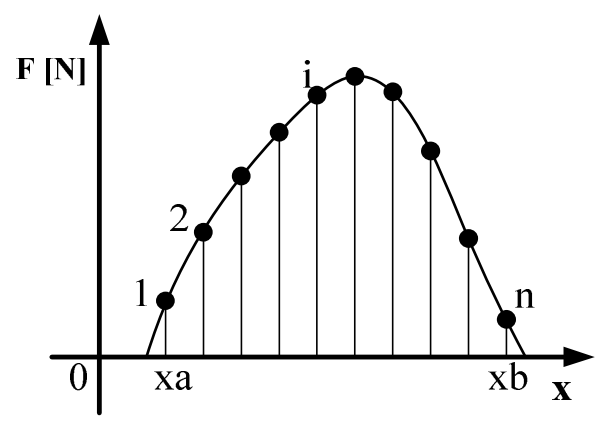

Fig. 8. The determination of the average force value

The average force value of the concerned interval can be sufficiently approximated by

$$
\hat{F}=\frac{\int_{a}^{b} F(x) d x}{b-a} \cong \frac{\sum_{i=1}^{n} F_{i}}{n} .
$$

The maximum value of the force function is determined by

$$
F_{\max }=\max _{i}\left\{F_{i} \geq \delta_{3}\right\} \text {, where } \delta_{3}>0 \text { a prescribed value. }
$$


The slope $\tan \alpha_{i}$ can be formulated as

$$
\tan \alpha_{i}=\frac{F_{i}-F_{i-k}}{k}, \quad \text { where } \quad k \geq 1
$$

Furthermore, a nominal average force $\hat{F}_{\text {nom }}$ and a nominal maximum force $F_{\text {max }}^{\text {nom }}$ values are stored in the MC, which were determined by a previous experiment.

The decision is made by the fulfillment of the problem dependent combination of four conditions, which are given by

$$
\left\{\frac{\left|\hat{F}_{\text {nom }}-\hat{F}\right|}{\hat{F}_{\text {nom }}}<\delta_{1}\right\},\left\{\frac{\left|F_{\max }^{\text {nom }}-F_{\max }\right|}{F_{\max }^{n o m}}<\delta_{2}\right\},\left\{\tan \alpha_{i}<0\right\},\left\{F_{i}<\delta_{3}\right\} .
$$

In this paper the handling is terminated when the first and the last conditions of (15) with logical relational operator AND provided true.

\subsection{Measurements}

In order to validate the simulation a test measurement is performed. The measurement of the closing operation with the simulation results are shown in Fig. 9. The obtained fine solid curve represents the simulation results with characteristic points. The result of the measurement is indicated by a thick solid curve, its characteristic points are denoted by black circles. The maximum occurring closing force is $F_{\text {max }}$.

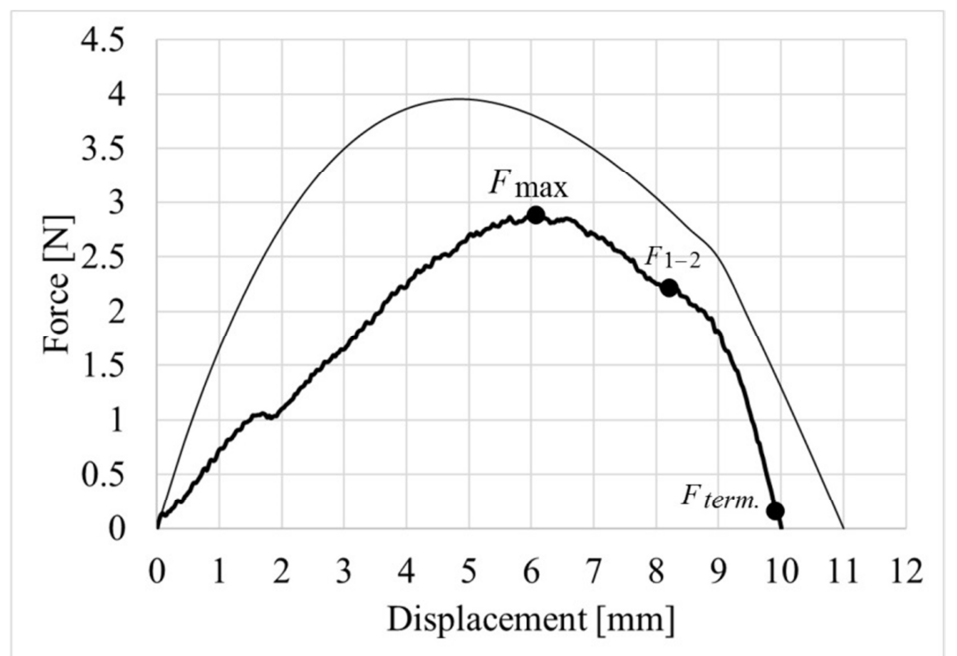

Fig. 9. The measurement with the simulation results 
On the downhill part of the load-deflection curve at point $F_{1-2}$ there is a sharp change in the gradient due to contact of the buckle (see point $\mathrm{C}$ shown in Fig. 2). The location of the termination point is denoted by $F_{\text {term }}$, which is higher than zero since the assembly performed dynamically.

The two curves in Fig. 9 are comparable but it shows that the stiffness of the simulation model is higher than the real structure, since the real joints possess backlashes.

\section{Conclusion}

Snap-fit modeling and robotic handling of a plastic box buckle have been discussed in this paper. Nonlinear large displacements with small strains were used to treat the modeling and simulation processes. The difference between the geometric stiffness matrices obtained by consistent and co-rotational methods provided similar number of iterations noting that, the former performed better. A self-devised intelligent endeffector is capable to recognize snap-fit phenomenon and terminate automatically the handling process. The end effector can be used also to measure the whole snap-fit process and save the data to a computer.

The simulation and the real measurements show differences, due to the back-lash property of the joints of the buckle.

\section{Acknowledgements}

The described article was carried out as part of the EFOP-3.6.1-16-2016-00011 'Younger and Renewing University - Innovative Knowledge City - institutional development of the University of Miskolc aiming at intelligent specialization' project implemented in the framework of the Szechenyi 2020 program. The realization of this project is supported by the European Union, co-financed by the European Social Fund.

\section{Open Access statement}

This is an open-access article distributed under the terms of the Creative Commons Attribution 4.0 International License (https://creativecommons.org/licenses/by/4.0/), which permits unrestricted use, distribution, and reproduction in any medium, provided the original author and source are credited, a link to the CC License is provided, and changes - if any - are indicated. (SID_1)

\section{References}

[1] Radi M., Reinhart G. Industrial haptic robot, Guidance system for assembly processes, IEEE International Workshop on Haptic Audio visual Environments and Games, Lecco, Italy, 7-8 November 2009, pp. 69-74. 
[2] Newman W. S., Branicky M. S., Podgurski H. A., Chhatpar S., Swaminathan L. Huang, J., Zhang $\mathrm{H}$. Force-responsive robotic assembly of transmission components, IEEE International Conference on Robotics \& Automation, Detroit, USA, 10-15 May 1999, pp. 2096-2102.

[3] Loske J., Biesenbach R. Force-torque sensor integration in industrial robot control, 15th International Workshop on Research and Education in Mechatronics, El Gouna, Egypt, 9-11 September 2014, pp. 1-5.

[4] Bayer Material Science LLC. Snap-Fit Joints for Plastics - A Design Guide, Bayer Polycarbonate Business Unit, Pittsburg, PA, 1998.

[5] Rónai L., Szabó T. Snap-fit assembly process with industrial robot including force feedback, Robotica, Cambridge University Press, Article in Press, Published online: 14 May 2019, pages 1-20.

[6] Ren C., Gong Y., Jia F., Wang X. Theoretical analysis of a six-axis force/torque sensor with overload protection for polishing robot, 23rd International Conference on Mechatronics and Machine Vision in Practice, Nanjing, China, 28-30 November 2016, pp. 1-6.

[7] Liu J., Li P., Li Z. A multi-sensory end-effector for spherical fruit harvesting robot, IEEE International Conf. on Automation and Logistics, Jinan, China, 18-21 August 2007, pp. 258-262.

[8] Crisfield M. A. Non-linear finite element analysis of solids and structures, John Wiley and Sons, Chichester, UK, 1991.

[9] de Souza R. M. Force-based finite element for large displacement inelastic analysis of frames, $P h D$ Thesis, Department of Civil and Environmental Engineering, University of California, Berkeley, 2000.

[10] McGuire W., Gallagher R. H., Ziemian R. D. Matrix structural analysis, Second Edition, John Wiley and Sons, Printed in USA, 2000.

[11] Rónai L. Design aspects of a robotic end-effector, Design of Machines and Structures, Vol. 8, No. 2, 2019, pp. 52-58. 